\title{
BLOOD VESSEL SEGMENTATION USING MOVING-WINDOW ROBUST AUTOMATIC THRESHOLD SELECTION
}

\author{
Michael H. F. Wilkinson, Tsjipke Wijbenga, Gijs de Vries, and Michel A. Westenberg \\ Institute of Mathematics and Computing Science, University of Groningen \\ P.O. Box 800, 9700 AV, Groningen, The Netherlands
}

\begin{abstract}
Two moving-window methods, using either flat or Gaussian weighted windows, for local thresholding with Robust Automatic Threshold Selection are developed. The results show that fast segmentation of blood vessels against a varying background and noise is possible at modest computational cost. Volumes of $128 \times 256^{2}$ and $256^{3}$ can be segmented in $3.1 \mathrm{~s}$ and $6.6 \mathrm{~s}$, for flat, and $12.6 \mathrm{~s}$ and $30.8 \mathrm{~s}$ for Gaussian windows, respectively, on a $1.9 \mathrm{GHz}$ Pentium 4.
\end{abstract}

\section{INTRODUCTION}

Thresholding is still one of the most commonly used methods of image segmentation. Apart from its use as a standalone segmentation method, thresholding may yield a good initial estimate from which more advanced methods can proceed $[1,2]$. In all applications of thresholding, correct selection of the threshold is the key issue, and many methods for automatic selection of optimal thresholds have been published [3, 4]. Local thresholding can deal properly with a locally varying background, or variations in the grey level of objects, both of which may occur in a single image [4].

In this paper we will focus on a particular local, bilevel thresholding method, called Robust Automatic Threshold Selection (RATS) [5]. Despite its successful application to 2-D microscopic images, [6], it has never been adapted to 3$\mathrm{D}$ medical images. We will develop new, moving-window versions of the algorithm and apply them to segmentation of blood vessels in 3-D angiograms. We will compare the results with those of earlier tiled local thresholding with RATS [5, 6], and with those obtained with the simple percentile thresholding preprocessing step used for vessel segmentation used by several authors $[1,2]$. The use of percentile thresholders has two problems: (i) they are not adaptive to local changes in image properties, and (ii) choosing the correct percentile is done heuristically, so the results for, e.g., abdominal angiograms are of no use in, e.g., cerebral angiography. In the former the 95th percentile is recommended by [2]. However, on a cerebral magnetic resonance angiogram (MRA) we found that the 99th yielded considerably better results, as can be seen in Figure 1.

\section{ROBUST AUTOMATIC THRESHOLD SELECTION}

RATS [5] is a simple and fast method for bilevel thresholding of grey scale images. Kittler et al. [5] show that the optimal threshold $T$ in a noise-free image is given by

$$
T=\frac{\sum e(x, y) p(x, y)}{\sum e(x, y)},
$$

in which $p(x, y)$ is the grey level at $(x, y)$ and the edge strength $e(x, y)$ is given by

$$
e(x, y)=\max \left(g_{x}(x, y), g_{y}(x, y)\right)
$$

with

$$
g_{x}(x, y)=|p(x-1, y)-p(x+1, y)|
$$

and

$$
g_{y}(x, y)=|p(x, y-1)-p(x, y+1)| \text {. }
$$

In the presence of noise, $T$ is biased towards the most common category in the image. This noise bias is counteracted by using a threshold on the edge strength below which the pixels are not weighted at all. The statistic now becomes

$$
T=\frac{\sum w(x, y) p(x, y)}{\sum w(x, y)}
$$

with

$$
w(x, y)= \begin{cases}e(x, y) & \text { if } e(x, y)>\lambda \eta \\ 0 & \text { otherwise }\end{cases}
$$

in which $\eta$ is the standard deviation of the image noise, and $\lambda$ is an adjustable parameter, which depends on the actual edge strength used. For the edge strength defined in (2) it was shown empirically that $\lambda=5$ is a good choice for Gaussian noise [7].

It has been shown that any edge detector with an even response to a step edge at the origin will yield the same 


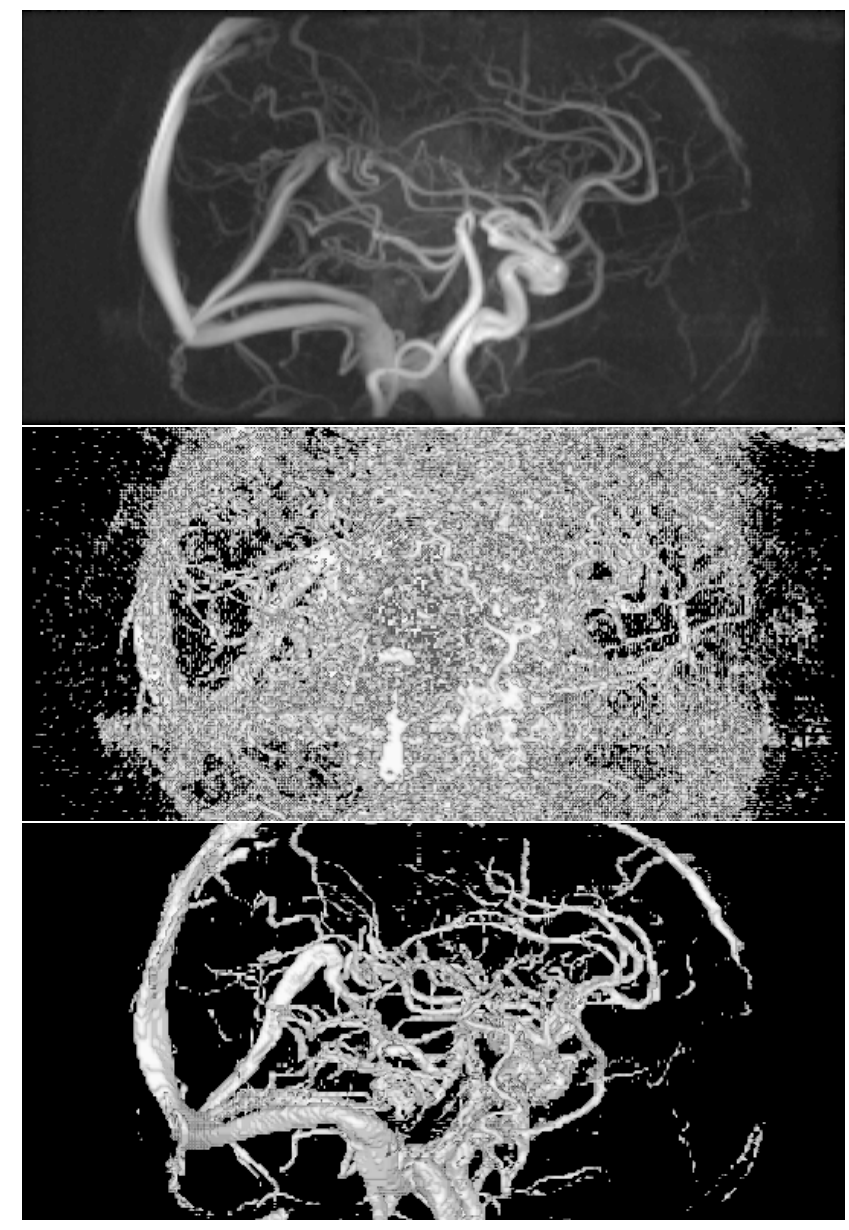

Fig. 1. Thresholding an MRA of a human brain: (top) MIP of original; surface renderings of (middle) 95th percentile thresholded result, and (bottom) 99th percentile thresholded result. The middle result is practically useless.

optimal $T$ in any number of dimensions [7]. In particular, the gradient detector

$$
g^{2}(x, y, z)=g_{x}^{2}(x, y, z)+g_{y}^{2}(x, y, z)+g_{z}^{2}(x, y, z)
$$

with $g_{x}, g_{y}$, and $g_{z}$ defined in an analogous way as in (3) and (4), shows no curvature bias and is rotation invariant. Besides, it has a reduced noise bias, as was already noted by Kahmoun and Astruc in 1-D [8]. However, the reduced noise bias comes at the expense of increased variance, which can be countered by using Sobel filter kernels to compute $x$, $y$, and $z$ derivatives, in which case $\lambda=3$ is recommended [7]. This value will be used throughout this paper.

\subsection{Local Application of RATS}

RATS lends itself well to local application [5] for two reasons: (i) the statistic in (5) is robust against noise, and (ii) it is easy to check whether a region contains an edge by checking whether the denominator in (5) is above some threshold $[5,7]$. Two strategies for local application of RATS have been developed $[5,6]$. These methods divide the image into non-overlapping rectangular regions (of in the order of $32 \times 32$ pixels), compute $T$ if possible and assign the threshold to the center pixel of the region. Once all thresholds have been assigned to the central pixels of each region, all other pixels are assigned thresholds by bi-linear interpolation.

A problem arises from the fact that the threshold is applied at the center of the region, whereas the edge which generated the statistic may be to one side of the region. This is demonstrated in Figure 2. The threshold surface will not in general be at the right level at the edges themselves, i.e., where they matter most. When using a moving window approach, the threshold applied at each edge pixel is computed from an area centered on the edge pixel itself, reducing this effect.

\subsection{Moving Window RATS}

The threshold surface computed by a moving window version of RATS can be written as

$$
T(x, y, z)=\frac{\sum_{i=x-h}^{x+h} \sum_{j=y-h}^{y+h} \sum_{k=z-h}^{z+h} w(i, j, k) p(i, j, k)}{\sum_{i=x-h}^{x+h} \sum_{j=y-h}^{y+h} \sum_{k=z-h}^{z+h} w(i, j, k)},
$$

which can be written as a the ratio of two convolutions

$$
T_{h}(x, y, z)=\frac{\left(\Pi_{h} *(w \cdot p)\right)(x, y, z)}{\left(\Pi_{h} * w\right)(x, y, z)},
$$

in which $*$ denotes convolution and $\Pi_{h}(x, y, z)$ is given by

$$
\Pi_{h}(x, y, z)= \begin{cases}1 & \text { if }|x| \leq h,|y| \leq h, \text { and }|z| \leq h \\ 0 & \text { otherwise }\end{cases}
$$

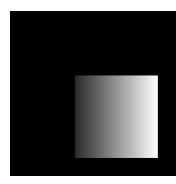

(a)

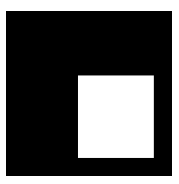

(b)

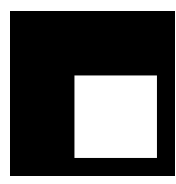

(c)

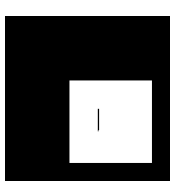

(d)
Fig. 2. Problems with tiled local RATS: (a) image of shaded square on uniform background; (b) using a two level quadtree (four leaves), the threshold at the left-hand side of the square is overestimated; (c) using a 3 level quad-tree correct segmentation is achieved, but with (d) 4 levels an artefact appears inside the square. 
One problem with (9) is that $T$ is undefined for all voxels where $\left(\Pi_{h} * w\right)(x, y, z)=0$. However, the convolution formalism allows generalization of the algorithm to other convolution kernels, e.g. Gaussian. Gaussian kernels are rotation invariant and have infinite impulse response (IIR), and so will contribute over the entire image. Besides, they are separable, and can be computed quickly using a recursive implementation, which has an IIR [9]. We arrive at

$$
T_{\sigma}(x, y)=\frac{\left(G_{\sigma} *(w \cdot p)\right)(x, y)}{\left(G_{\sigma} * w\right)(x, y)},
$$

with $G_{\sigma}$ a Gaussian with zero mean and standard deviation of $\sigma$. A further advantage of (11) over (9) is that edges close to the current pixel are given higher weights than distant edges.

\section{APPLICATION TO ANGIOGRAMS}

We implemented oct-tree-based RATS (OT-RATS) [6], moving window RATS (MW-RATS), and the Gaussian variant (GW-RATS) in 3-D. When using MW-RATS according to (9) pixels were set to background whenever the denominator was zero. This is safe as long as the width of the window exceeds the width of the thickest vessels. However, choosing a narrow window improves the localization of the threshold computation, which could lead to spurious holes in foreground objects. Using the knowledge that holes in vessels are impossible, we chose a narrow window, but used post-processing to remove holes, which are connected background components which do not touch the image border. This was done using the union-find method [10].

A comparison of OT-RATS, MW-RATS, and GW-RATS is given in Figure 3. We use the same edge threshold level $\lambda \eta$ and for OT-RATS and MW-RATS approximately the same size of the region used to determine the thresholds (2048 and $13^{3}=2197$ respectively). In the case of GWRATS we chose $\sigma=4$ in this comparison, so that a sphere of radius $2 \sigma$ has approximately the same volume (2145 voxels). OT-RATS produces a number of annoying artefacts, some of which are clearly visible as somewhat amorphous clumps in the top image. This is lacking in the lower two images in Figure 3. The GW-RATS result appears to be cleaner than that of MW-RATS. In particular, the middle image of Figure 3 shows a kind of "halo" of noise responses around certain larger vessels (conspicuous on the lefthand side). Besides, the area on the lower lefthand side of the image, just below a large vessel contains various almost linear artefacts in the MW-RATS result, which are absent in both the OT-RATS and GW-RATS results.

In Figure 4 the sensitivity of the method to the estimate of $\eta$ is explored. At $\eta=10$ the result is unusable; at $\eta=40$ small vessels disappear, but the large vessels remain unchanged. Vessel enhancement filtering using attribute filters

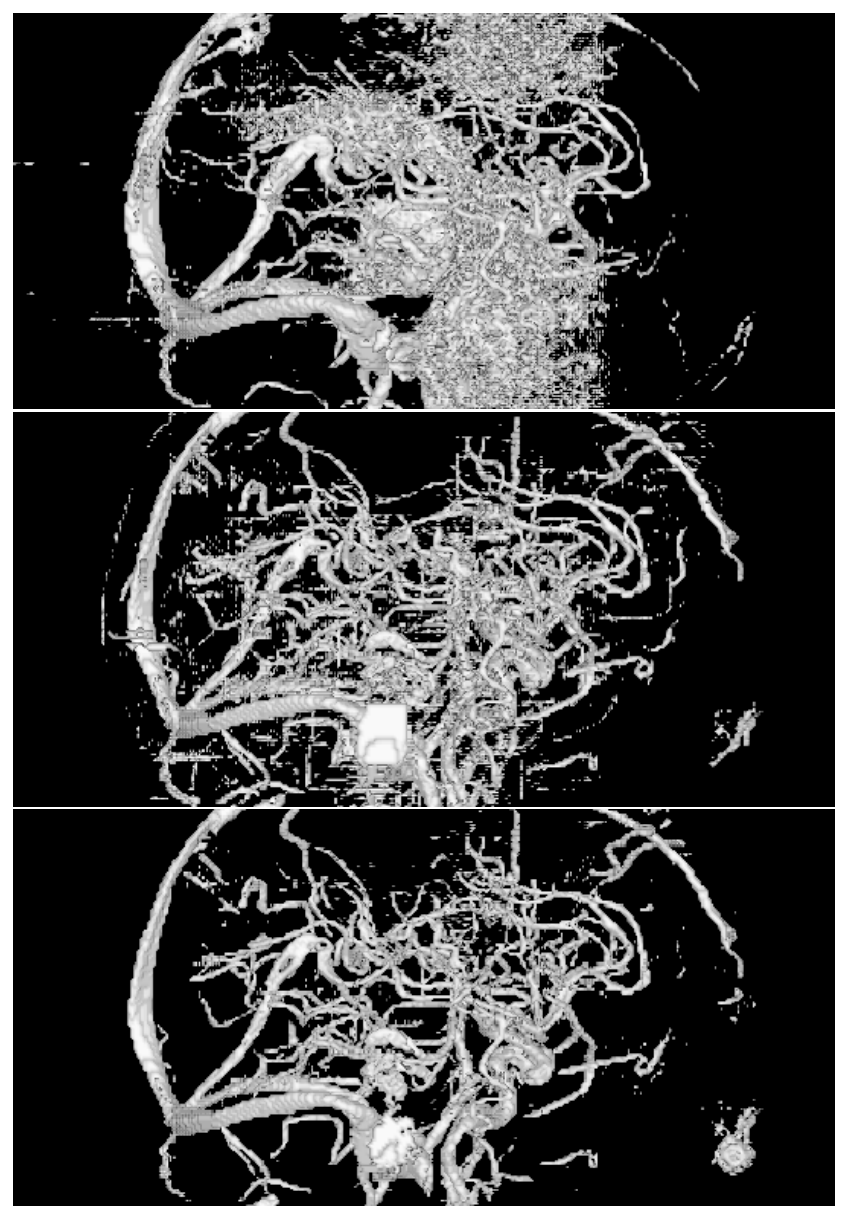

Fig. 3. Thresholding same MRA of a human brain as in Fig. 1 using $\lambda=3$ and $\eta=25$ : surface renderings of (top) OT-RATS result with 5-level oct-tree; (middle) MW-RATS result using window $13^{3}$ voxels; and (bottom) GW-RATS result with $\sigma=4$.

from [11] greatly reduces noise, whilst retaining the vessels, allowing filtering with $\eta=1$ on the processed image.

MW-RATS is fast, requiring only $3.1 \mathrm{~s}$ of computing time for a $128 \times 256^{2}$ volume, and $6.6 \mathrm{~s}$ for a $256^{3}$ volume on a $1.9 \mathrm{GHz}$ Pentium 4 based PC with $512 \mathrm{MB}$ of $800 \mathrm{MHz}$ RDRAM. GW-RATS is slower, requiring $12.6 \mathrm{~s}$ and $30.8 \mathrm{~s}$ for the same two volumes respectively.

\section{DISCUSSION}

The new moving window versions of RATS, and in particular GW-RATS, show a good deal of promise in segmenting vascular structures in 3-D angiograms. The number of tunable parameters is fairly low: window size (or $\sigma$ ) and $\lambda$. Parameter $\eta$ should be an estimate for the image noise and could be determined experimentally, possibly from the im- 


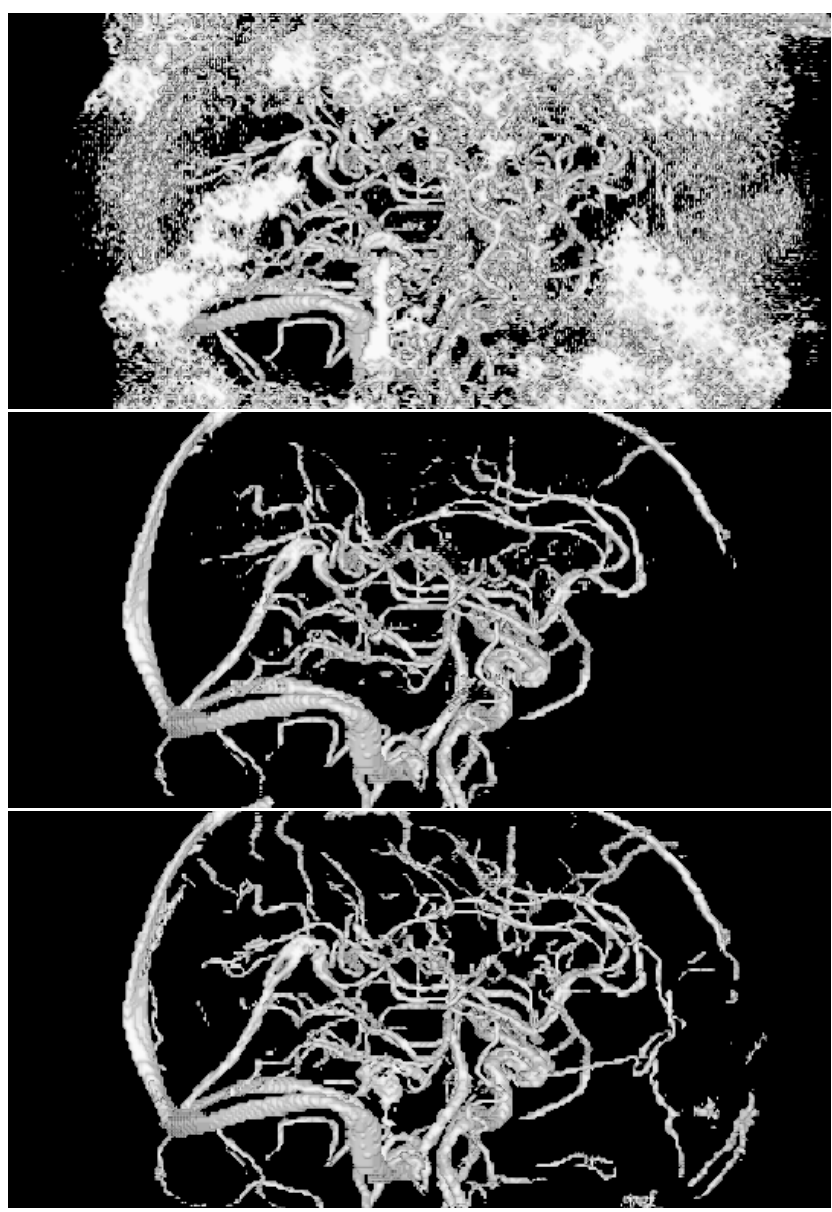

Fig. 4. Surface rendering of GW-RATS result of same angiogram as in Fig. 1 using $\sigma=4$ and (top) $\eta=10$; (middle) $\eta=40$; (bottom) $\eta=1$ after filtering the input image with the vessel enhancement filter in [11].

age itself as suggested in [5]. Further extensions of the moving window methods might include multi-scale versions, in which if a threshold cannot be assigned at a small scale, runs at progressively larger window sizes are performed to classify any unclassified pixels. Because both convolutions have a cost independent of the window size, the cost of the multi-scale version is proportional to the number of scales.

More work is needed to provide a full evaluation of the method, both as a stand-alone segmentation method and as a preprocessing step for other, more advanced methods. In particular a larger number of angiograms must be processed and the results reviewed by human experts. Besides, phantoms for which the ground truth segmentation is known must be used to give objective estimates of segmentation performance. Such an extended study is now under way. However, the results show that MW-RATS and GW-RATS can segment complex images with filamentous structures at modest computational cost. Other possible applications include filamentous microorganisms such as fungi, or text.

\section{REFERENCES}

[1] Y. Masutani, T. Schiemann, and K.-H. Höhne, "Vascular shape segmentation and structure extraction using a shape based region-growing model," in Proc. MICCAI'98, 1998, vol. 1496 of Lecture Notes in Computer Science, pp. 1242-1249.

[2] R. M. Stefancik and M. Sonka, "Highly automated segmentation of arterial and venous trees from three-dimensional magnetic resonance angiography (MRA)," Int. J. Cardiovasc. Imaging., vol. 17, pp. 3747, 2001.

[3] Ø. D. Trier and A. K. Jain, "Goal-directed evalution of binarization methods," IEEE Trans. Image Proc., vol. 17, no. 12, pp. 1191-1201, 1995.

[4] Y. Yang and H. Yan, "An adaptive logical method for binarization of degraded document images," Pattern Recognition, vol. 33, pp. 787-807, 2000.

[5] J. Kittler, J. Illingworth, and J. Föglein, "Threshold selection based on a simple image statistic," Comp. Vision Graph. Image Proc., vol. 30, pp. 125-147, 1985.

[6] M. H. F. Wilkinson, "Rapid automatic segmentation of fluorescent and phase-contrast images of bacteria," in Fluorescence Microscopy and Fluorescent Probes, J. Slavik, Ed., pp. 261-266. Plenum Press, New York, 1996.

[7] M. H. F. Wilkinson, "Optimizing edge detectors for robust automatic threshold selection: coping with edge curvature and noise," Graph. Mod. Image Proc., vol. 60, pp. 385-401, 1998.

[8] F. Kahmoun and J. P. Astruc, "Optimum edge detection for object-background segmentation," CVGIP: Graph. Mod. Image Proc., vol. 56, pp. 25-28, 1994.

[9] I. T. Young and L. J. van Vliet, "Recursive implementation of the Gaussian filter," Signal Processing, vol. 44, pp. 139-151, 1995.

[10] M.B. Dillencourt, H. Samet, and M. Tamminen, "A general approach to connected-component labeling for arbitrary image representations," J. ACM, vol. 39, pp. 253-280, 1992.

[11] M. H. F. Wilkinson and M. A. Westenberg, "Shape preserving filament enhancement filtering," in Proc. MICCAI'2001, W. J. Niessen and M. A. Viergever, Eds., 2001, vol. 2208 of Lecture Notes in Computer Science, pp. 770-777. 